\title{
Wideband Receive Spatial Modulation with Time Domain Pre-Equalizer for Large MIMO Systems
}

\author{
Ahmed Raafat, Adrian Agustin and Josep Vidal \\ Dept. of Signal Theory and Communications, Universitat Politecnica de Catalunya (UPC), Barcelona, Spain. \\ Email: \{ahmed.raafat, adrian.agustin, josep.vidal\}@upc.edu
}

\begin{abstract}
Receive spatial modulation (RSM) schemes are promising for massive multiple-input-multiple-output (MIMO) systems at millimeter wave (mmWave) bands because they require reduced complexity and low consumption hardware at the user terminal and exploit the receive spatial dimension to attain high spectral efficiency. To the best of our knowledge, these schemes have been developed for narrowband transmission. In this paper, we adapt RSM schemes for outdoor wideband mmWave massive MIMO systems. We consider the downlink of a single user system operating with single carrier RSM and design a low complexity time-domain finite impulse response pre-equalizer to combat the intersymbol interference caused by the wideband transmission, assuming imperfect channel knowledge. We show that receive antenna selection (RAS) is necessary for attaining high spectral efficiency and we suggest fast and efficient RAS algorithm. Simulation results show that the proposed RSM scheme achieves comparable spectral efficiency to the fully digital orthogonal frequency division multiplexing MIMO system with superior energy efficiency.
\end{abstract}

Index Terms-RSM, Wideband, mmWave, Large MIMO.

\section{INTRODUCTION}

The vast spectrum available in the millimeter wave (mmWave) frequency band permits leveraging of the wide bandwidths to attain high data rates for the $5 \mathrm{G}$ systems [1]-[2]. Massive number of antennas at the base station (BS) and at the user terminal (UT) are necessary to provide high beamforming gain to combat the severe path loss of mmWave propagation. However, fully digital (FD) multiple-input-multiple-output (MIMO) suffers from expensive and power consuming devices at mmWave massive antennas systems.

Several wideband precoding schemes have been developed based on low complexity MIMO transceiver architectures. Wideband hybrid precoding was developed to reduce number of the radio-frequency (RF) chains and high resolution analog-to-digital-converters (ADCs) by adding analog energy efficient devices like phase shifters, power splitters and power combiners

The research leading to these results has been partially funded by the 5Gwireless project within the framework of H2020 Marie Skłodowska-Curie innovative training networks (ITNs), the project 5G\&B RUNNER-UPC (TEC2016-77148-C2-1-R (AEI/FEDER, UE)) and the Catalan Government (2017 SGR 578-AGAUR).
[3]. However, hybrid MIMO transceiver should comprise large number of analog devices to achieve high spectral efficiency (SE) and hence the hardware power consumption becomes significant [4].

Receive spatial modulation (RSM) schemes exploit the receive antenna as an additional dimension for data transmission [5]-[6] and allow energy efficient hardware design at the UT. An RSM scheme based on a single RF chain and one high resolution ADC is reported in [7] that attains high data rates at outdoor narrowband massive MIMO systems. In this paper, we adapt the energy efficient RSM scheme in [7] for outdoor wideband mmWave massive MIMO systems.

Orthogonal-frequency-division-multiplexing (OFDM) and single carrier with channel equalization are two methods to combat the frequency selectivity of the wideband transmission. In RSM schemes [5]-[7], it is possible to transmit one spatial symbol per channel use, thus symbols with shorter duration (single carrier) allow transmitting more spatial symbols and thus attaining higher SE. We propose a wideband single carrier RSM scheme based on a time-domain finite impulse response (FIR) pre-equalizer [8] and receive antenna selection (RAS). The contribution of this paper is as follows

- We propose a wideband single carrier RSM scheme based on a low complexity zero forcing (ZF) time-domain FIR pre-equalizer with optimized delay for the UT narrowband architecture in [7] and no channel state information (CSI) at the UT and imperfect CSI at the BS.

- We study the impact of the channel angular spread and the number of delay tap channels on the minimum number of FIR taps.

- We show that we can attain high SE by applying RAS and we provide fast and efficient RAS sequential algorithm based on QR decomposition [9].

\section{System AND Channel Models}

We consider the downlink (DL) of a large MIMO single user operating in the outdoor wideband mmWave propagation environment. The BS and the UT are 


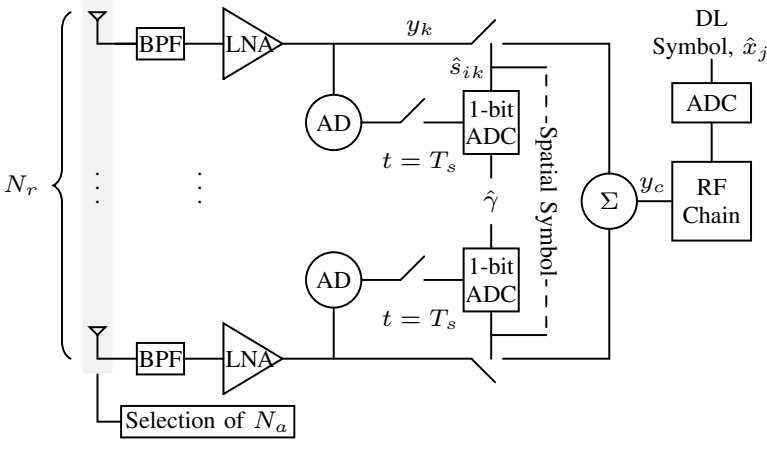

Fig. 1. Wideband RSM Large MIMO transceiver architecture [7]

equipped with $N_{t}$ and $N_{r}$ antennas, respectively. We consider energy efficient UT architecture (shown in Fig. 1 [7]) and low complexity pre-equalizer design that further illustrated in the sequel.

\section{A. Frequency selective channel model}

The severe path loss of mmWave propagation leads to limited scattering environment [10], so an accurate wideband channel model [11] is used in evaluating the proposed system performance. According to this model, the time domain delay-d MIMO channel matrix can be expressed as

$\mathbf{H}[d]=\sqrt{\frac{N_{t} N_{r}}{C L}} \sum_{i=1}^{C} \sum_{l=1}^{L} g_{l i} \mathrm{P}_{r c}\left(d T_{s}-\tau_{l i}\right) \mathbf{v}_{r}\left(\theta_{l i}\right) \mathbf{v}_{t}\left(\phi_{l i}\right)^{H}$

where $\mathbf{H}[d] \in \mathbb{C}^{N_{r} \times N_{t}}, d=0, \cdots, N_{c}-1, C$ is the number of scattering clusters and each cluster contributes with $L$ rays. Each ray has delay $\tau_{l i}$, complex gain $g_{l i}$, sampling time $T_{s}$ and azimuth and elevation angles of departures and arrivals $\left(\phi_{l i}, \theta_{l i}\right)$, respectively. We consider uniform linear array for the transmit and the receive array response vectors $\left(\mathbf{v}_{t}\left(\phi_{l i}\right), \mathbf{v}_{r}\left(\theta_{l i}\right)\right)$ where the $N$-antennas array response vector can be expressed as

$$
\mathbf{v}(\phi)=\frac{1}{\sqrt{N}}\left[1, e^{j k d_{s} \sin (\phi)}, \ldots, e^{j(N-1) k d_{s} \sin (\phi)}\right]^{T}
$$

where $d_{s}$ is the antennas spacing and $k=\frac{2 \pi}{\lambda}$. The raised-cosine pulse shaping filter $\mathrm{P}_{r c}(t)$ can be expressed as

$$
\mathrm{P}_{r c}(t)= \begin{cases}\frac{\pi}{4} \operatorname{sinc}\left(\frac{1}{2 \beta}\right), & \text { if } t= \pm \frac{T_{s}}{2 \beta} \\ \operatorname{sinc}\left(\frac{t}{T_{s}}\right) \frac{\cos \left(\frac{\pi \beta t}{T_{s}}\right)}{1-\left(\frac{2 \beta t}{T_{s}}\right)^{2}}, & \text { else, }\end{cases}
$$

In section $\mathrm{V}$ we show that RAS is necessary for the proposed RSM scheme to attain high SE. In the sequel, the matrix $\mathbf{H}_{a}[d]$ denotes the delay- $d$ tap channel after selecting $N_{a}$ antennas to be active at the UT.

For comparison purposes of the proposed energy efficient single carrier RSM scheme with FD $K$-subcarriers
OFDM MIMO, the channel matrix of the $k^{\text {th }}$ subcarrier can be obtained by applying (Discrete Fourier Transform) as follows

$$
\mathbf{H}_{f}[k]=\sum_{d=0}^{N_{c}-1} \mathbf{H}[d] e^{-j \frac{2 \pi k}{K} d}
$$

\section{B. System assumptions}

We exploit the channel reciprocity by considering time-division-duplex (TDD) transmission protocol where the CSI is needed only at the BS. The TDD frame is designed as follows, at first the UT transmits training symbols so that the BS can acquire the CSI. This can be achieved with low training overhead using sparse time domain channel estimation [11]. Then, the BS transmits few pilot symbols to enhance the quality of detection at the UT as detailed in section IV.

\section{Bit mapping strategy}

For the sake of designing an energy efficient UT architecture, we consider a single RF chain at the UT and thus the BS can transmit one modulation symbol per channel use $\left(x_{j} \in M-\right.$ ary constellation, $\left.j=1, \cdots, \log _{2} M\right)$. For attaining high SE, we exploit the large number of receive antennas by transmitting superimposed spatial symbol $\left(\mathbf{s}_{i} \in \mathbb{R}^{N_{a} \times 1}, i=\right.$ $1, \cdots, 2^{N_{a}}, N_{a} \leq N_{r}$ ) that includes $N_{a}$ data bits and can be detected using energy efficient hardware. The transmit vector at the precoder input at time $n, \mathbf{z}[n]$, can be expressed as

$$
\mathbf{z}[n]=\left\{\begin{array}{lll}
\mathbf{s}_{i}[n] x_{j}[n] & \text { if } & \mathbf{s}_{i}[n] \neq \mathbf{0}_{N_{r}} \\
\mathbf{s}_{i}[n] & \text { if } & \mathbf{s}_{i}[n]=\mathbf{0}_{N_{r}}
\end{array}\right.
$$

where $\mathrm{E}\left[\mathbf{z}[n] \mathbf{z}[n]^{H}\right] \approx \frac{1}{2} \mathbf{I}_{N_{a}}$.

\section{Energy efficient UT circuitry}

The UT architecture in Fig. 1 comprises one of the power consuming devices (RF chain, high resolution ADC) to detect the modulation symbol and $N_{r}$ energy efficient devices (amplitude detector (AD), 1-bit ADC) to detect the spatial symbol [7]. The AD detects the amplitude of the wideband RF signal with large input impedance and negligible power consumption [12].

\section{E. Low complexity FIR pre-equalization at the BS}

We consider ZF time-domain FIR pre-equalizer [8] with imperfect CSI at the BS and $L_{f}$ taps that converts the multiple delay taps channel into single tap channel to mitigate the intersymbol interference resulting from the delay spread. The proposed pre-equalizer does not require cyclic prefix or zero padding that saves transmission power and enhances the SE. In the sequel, we provide detailed analysis of the ZF pre-equalizer design with optimized delay assuming imperfect CSI. 
III. TIME DOMAIN ZF PRE-EQUALIZATION We consider pre-equalizer with $L_{f}$ taps $(\mathbf{F}[k] \in$ $\left.\mathbb{C}^{N_{t} \times N_{a}}, k=0, \cdots, L_{f}-1\right)$ such that the transmit precoded vector at time $n, \mathbf{x}[n]$, can be expressed as

$$
\mathbf{x}[n]=\sqrt{\alpha P_{t}} \sum_{k=0}^{L_{f}-1} \mathbf{F}[k] \mathbf{z}[n-k]
$$

where the normalization factor $\alpha \approx$ $2\left(\sum_{k=0}^{L_{f}-1}\|\mathbf{F}[k]\|_{F}^{2}\right)^{-1}, \quad P_{t}$ is the transmit power and the $\|\cdot\|_{F}$ denotes the Frobenius norm operator. The received vector at time $n$ can be expressed as

$$
\mathbf{y}[n]=\sum_{i=0}^{N_{c}-1} \mathbf{H}_{a}[i] \mathbf{x}[n-i]+\mathbf{n}[n]
$$

where the generated noise $\mathbf{n}[n] \in \mathbb{C}^{N_{a} \times 1}$ has independent and identically distributed (i.i.d) $\mathcal{C N}\left(0, \sigma^{2}\right)$ coefficients.

\section{A. Pre-equalizer design}

We design the ZF pre-equalizer to null the intersymbol interference such that the equalized channel $\left(\mathbf{H}_{e q}[n]\right)$ resulting from the convolution between the pre-equalizer and the time-domain channel taps is identity matrix at time $n_{d}$ and zero elsewhere as illustrated in equation (8).

$$
\mathbf{H}_{e q}[n]=\sum_{i=0}^{N_{c}-1} \mathbf{H}_{a}[i] \mathbf{F}[n-i]=\mathbf{I}_{N_{a}} \delta\left[n-n_{d}\right]
$$

where the delay $n_{d}$ is designed to maximize the received power. From equations (6)-(7) and (8), the received vector at time $n$ can be expressed as

$$
\begin{aligned}
\mathbf{y}[n] & =\sqrt{\alpha P_{t}} \sum_{i=0}^{N_{c}-1} \mathbf{H}_{e q}[i] \mathbf{z}[n-i]+\mathbf{n}[n] \\
& =\sqrt{\alpha P_{t}} \mathbf{z}\left[n-n_{d}\right]+\mathbf{n}[n]
\end{aligned}
$$

To design the pre-equalizer, we write equation (8) as

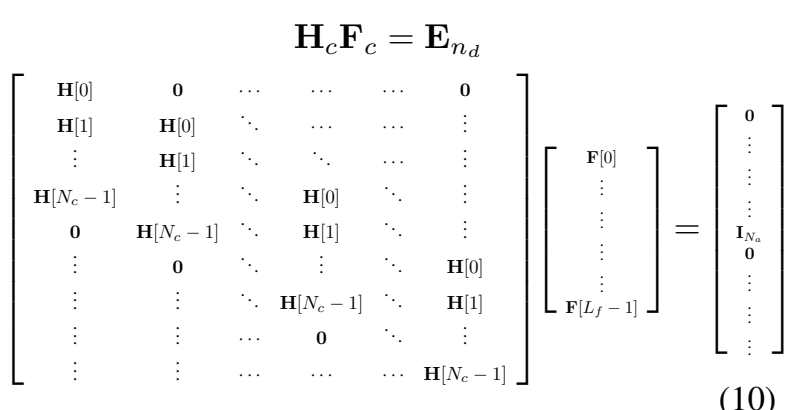

where $\mathbf{H}_{c} \in \mathbb{C}^{\left(N_{c}+L_{f}-1\right) N_{a} \times L_{f} N_{t}}, \mathbf{F}_{c} \in \mathbb{C}^{L_{f} N_{t} \times N_{a}}$ and $\mathbf{E}_{n_{d}} \in \mathbb{C}^{\left(N_{c}+L_{f}-1\right) N_{a} \times N_{a}}$. From equation (10), the $\mathrm{ZF}$ pre-equalizer can be expressed as

$$
\mathbf{F}_{c}=\mathbf{H}_{c}^{H}\left(\mathbf{H}_{c} \mathbf{H}_{c}^{H}\right)^{-1} \mathbf{E}_{n_{d}}
$$

where the position of the identity matrix in $\mathbf{E}_{n d}$ means that the signal will be received after $\left(0 \leq n_{d} \leq\right.$ $\left.N_{c}+L_{f}-2\right)$ time lags. We optimise this position so as to maximize the receive power (minimize the $\left.\sum_{k=0}^{L_{f}-1}\|\mathbf{F}[k]\|_{F}^{2}\right)$.

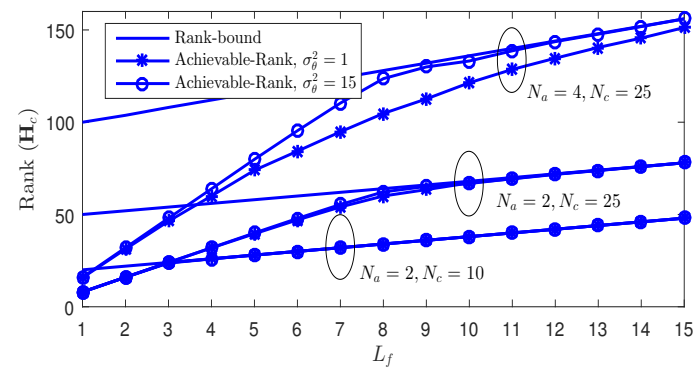

Fig. 2. Achievable rank and Rank-bound with $L_{f}$ at $N_{r}=$ $16, N_{t}=128$ and (average over 1000 channel realizations).

\section{B. Analysis of number of pre-equalizer taps}

The realization of the pseudoinverse as in equation (11) implies the following necessary and sufficient condition:

$$
\operatorname{Rank}\left(\mathbf{H}_{c}\right)=\left(N_{c}+L_{f}-1\right) N_{a}
$$

which can be achieved by ensuring the necessary condition:

$$
L_{f} N_{t} \geqslant\left(N_{c}+L_{f}-1\right) N_{a}
$$

where there is a maximum number of active receive antennas (ARA) for a given $L_{f}$ and a minimum number of needed FIR taps for a particular $N_{a}$ relying on the channel angular and time spreads, respectively as illustrated in the sequel.

In Fig. 2, we show the effect of $L_{f}, N_{c}, N_{a}$ and the angular spread on the feasibility of the pseudoinverse in equation (11). By increasing $L_{f}$, the achievable rank that is, the actual rank of matrix $\mathbf{H}_{c}$ grows at a faster pace than the Rank-bound $\left(N_{c}+L_{f}-1\right) N_{a}$ and matches the Rank-bound at the minimum number of needed FIR taps. The time spread $N_{c}$ leads to boost of the Rank-bound and thus, more FIR taps are needed to allow the $\operatorname{Rank}\left(\mathbf{H}_{c}\right)$ reaching the Rankbound. Large $N_{a}$ requires sufficient angular spread to allow ZF of $N_{a}$ spatial streams spread in $N_{c}$ time slots; however, mmWave propagation suffers from narrow angular spread [2]. Thus, the smart reduction of $N_{a}$ by the RAS is vital to fulfill the conditions in equation (12).

\section{Imperfect CSI at the BS}

Let us consider that the channel taps are estimated in error: $\hat{\mathbf{H}}[d]=\mathbf{H}[d]+\underline{\mathbf{H}}_{e}[d]$ where $\underline{\mathbf{H}}_{e}[d]$ is error matrix has i.i.d $\mathcal{C N}\left(0, \sigma_{e}^{2}\right)$ entries. The pre-equalizer design based on imperfect CSI can be expressed as

$$
\hat{\mathbf{F}}_{c}=\left(\mathbf{H}_{c}+\mathbf{H}_{e}\right)^{\dagger} \mathbf{E}_{n_{d}}=\hat{\mathbf{H}}_{c}^{\dagger} \mathbf{E}_{n_{d}}
$$


where $\mathbf{H}_{e}$ is the convolution error matrix. The equalized channel matrix with CSI errors can be expressed as

$$
\begin{gathered}
{\left[\hat{\mathbf{H}}_{e q}[0], \cdots, \hat{\mathbf{H}}_{e q}\left[N_{c}+L_{f}-2\right]\right]^{T}=\mathbf{H}_{c} \hat{\mathbf{F}}_{c}} \\
=\left(\hat{\mathbf{H}}_{c}-\mathbf{H}_{e}\right) \hat{\mathbf{F}}_{c}=\mathbf{E}_{n_{d}}-\mathbf{H}_{e} \hat{\mathbf{F}}_{c} \\
\hat{\mathbf{H}}_{e q}[n]=\mathbf{I}_{N_{a}} \delta\left[n-n_{d}\right]-\sum_{j=0}^{N_{c}-1} \underline{\mathbf{H}}_{e}[j] \hat{\mathbf{F}} \quad[n-j] \quad(15)
\end{gathered}
$$

Equation (15) shows that the equalized channel is not only identity at time $n_{d}$ as equation (8) but it also suffers from several error delay tap channels and thus, we cannot ensure perfect $\mathrm{ZF}$. According to equation (15), the received signal with residual inter-symbol interference can be expressed as

$$
\begin{aligned}
\mathbf{y}[n] & =\sqrt{\hat{\alpha} P_{t}} \mathbf{z}\left[n-n_{d}\right]+\mathbf{n}[n]- \\
& \sqrt{\hat{\alpha} P_{t}} \sum_{i=0}^{N_{c}+L_{f}-2}\left(\sum_{j=0}^{N_{c}-1} \underline{\mathbf{H}}_{e}[j] \hat{\mathbf{F}}[i-j]\right) \mathbf{z}[n-i]
\end{aligned}
$$

where $\hat{\alpha}=2 /\left\|\hat{\mathbf{F}}_{c}\right\|_{F}^{2}$

According to equation (16), the covariance matrix (C) of the interference and the generated noise can be expressed as

$$
\begin{gathered}
\mathbf{C}=\sigma_{n}^{2} \mathbf{I}_{N_{a}}+ \\
\hat{\alpha} P_{t} \mathrm{E}\left[\left(\sum_{i=0}^{N_{c}+L_{f}-2}\left(\sum_{j=0}^{N_{c}-1} \underline{\mathbf{H}}_{e}[j] \hat{\mathbf{F}}[i-j]\right) \mathbf{z}[n-i]\right)\right. \\
\left.\times\left(\sum_{i=0}^{N_{c}+L_{f}-2}\left(\sum_{j=0}^{N_{c}-1} \underline{\mathbf{H}}_{e}[j] \hat{\mathbf{F}}[i-j]\right) \mathbf{z}[n-i]\right)^{H}\right] \\
=\left(\sigma_{n}^{2}+\frac{\hat{\alpha} P_{t}}{2} \operatorname{Tr}\{\mathbf{Q}\}\right) \mathbf{I}_{N_{a}}=\hat{\sigma}_{n}^{2} \mathbf{I}_{N_{a}}
\end{gathered}
$$

where $\mathbf{Q}$ is a block diagonal matrix with diagonal matrices $\left(\mathbf{Q}_{i i}, i=0, \cdots, N_{c}+L_{f}-2\right)$ in the $i^{\text {th }}$ block such that

$$
\mathbf{Q}_{i i}=\frac{\sigma_{e}^{2}}{N_{a}}\left(\sum_{j=0}^{N_{c}-1}\|\hat{\mathbf{F}}[i-j]\|_{F}^{2}\right) \mathbf{I}_{N_{a}}
$$

We design $n_{d}$ so as to maximize the received SINR as follows

$$
\begin{array}{r}
n_{d}=\arg \max _{n_{d}=i} \frac{\hat{\alpha} P_{t}}{\sigma_{n}^{2}+\frac{\hat{\alpha} P_{t}}{2} \operatorname{Tr}\{\mathbf{Q}\}}, \\
i=0, \cdots, N_{c}+L_{f}-2 .
\end{array}
$$

where the optimal $n_{d}$ can be obtained by exhaustive search over $\left(N_{c}+L_{f}-1\right)$ values.

\section{Detection}

In this section, we illustrate how the energy efficient hardware at the UT can be exploited to recover the spatial and the modulation symbols with no availability of CSI at the UT.

\section{A. Spatial symbol detection}

We consider maximum likelihood (ML) spatial symbol detector that can be implemented using energy efficient devices at the UT (AD, 1-bit ADC) assuming Gaussian intersymbol interference. In [7], the authors show that when applying ZF precoder the (joint or per antenna) ML spatial symbol detectors lead to the same results. The received signal per the $k^{\text {th }}$ ARA at time $n$ assuming imperfect CSI at the BS can be expressed as

$$
y_{k}[n]=\sqrt{\hat{\alpha} P_{t}} s_{i k}\left[n-n_{d}\right] x_{j}\left[n-n_{d}\right]+\hat{n}[n]
$$

where $s_{i k} \in\{0,1\}$ is the $k^{\text {th }}$ spatial bit and $\hat{n}$ is the noise plus the intersymbol interference. The signal provided by the $\mathrm{AD}$ connected to the $k^{\text {th }}$ ARA can be written as

$$
a_{k}[n]=\left|y_{k}[n]\right|
$$

The ML spatial symbol detector has been developed at equation (16) in [7] and can be expressed as

$$
\hat{s}_{i k}\left[n-n_{d}\right]=\left\{\begin{array}{ll}
1 & \text { if } a_{k}[n]>\gamma \\
0 & \text { if } a_{k}[n]<\gamma
\end{array}, \quad \gamma \approx \frac{1}{2} \sqrt{\hat{\alpha} P_{t}}\right.
$$

The threshold $\gamma$ is fixed during the channel coherence time and the UT needs to estimate $\gamma$ to detect the spatial symbol. In [7], the authors show that only one DL pilot symbol is enough to allow the UT estimates $\gamma$ efficiently.

\section{B. Modulation symbol detection}

We consider limited noise combining scheme to detect the modulation symbol using single RF chain and one high resolution ADC. In order not to accumulate noise, the $k^{\text {th }}$ detected spatial bit $\hat{s}_{i k}\left[n-n_{d}\right]$ controls switch such that $y_{k}[n]$ is allowed to pass through the RF chain only if $\hat{s}_{i k}\left[n-n_{d}\right]=1$. The combined signal, $y_{c}[n]$ (see Fig. 1), can be expressed as

$$
y_{c}[n]=\frac{1}{\sum_{k=1}^{N_{a}} \hat{s}_{i k}\left[n-n_{d}\right]} \sum_{k=1}^{N_{a}} \hat{s}_{i k}\left[n-n_{d}\right] y_{k}[n]
$$

\section{ACHIEVABle RATES AND RAS}

We consider the mutual information for comparison purposes of the proposed scheme with OFDM FD MIMO system. We show that the spatial and modulation symbols mutual information can be modeled using binary asymmetric channel and the multipleinput-single-output channel, respectively. By applying the mutual information chain rule [13] it is shown in [14] that the RSM mutual information can be written as

$$
I_{\mathrm{RSM}}=I_{s}+I_{m}
$$

where $I_{s}$ and $I_{m}$ are the achievable rates by the spatial and modulation symbols, respectively. 


\section{A. Spatial symbol achievable rate}

The $k^{\text {th }}$ ARA can detect spatial bit $\hat{s}_{i k}\left[n-n_{d}\right]=$ $s_{i k}\left[n-n_{d}\right]=1$ or 0 with probability $p_{1}$ or $p_{0}$, respectively. Thus, $I_{s}$ can be obtained using the binary asymmetric channel assumption [15] as

$I_{s}=N_{a}\left(\mathcal{H}\left(\frac{p_{1}+1-p_{0}}{2}\right)-\frac{\mathcal{H}\left(p_{1}\right)+\mathcal{H}\left(1-p_{0}\right)}{2}\right)$

where $\mathcal{H}(p)$ is the entropy function [13] and the probabilities $p_{1}$ and $p_{0}$ can be expressed as

$$
\begin{aligned}
p_{1} & =\operatorname{Pr}\left(a_{k}[n]>\gamma \mid s_{i k}\left[n-n_{d}\right]=1\right) \\
& =Q_{1}\left(\frac{1}{\hat{\sigma}_{n}} \sqrt{2 \hat{\alpha} P_{t}}, \frac{1}{\hat{\sigma}_{n}} \sqrt{2} \gamma\right) \\
p_{0} & =\operatorname{Pr}\left(a_{k}[n]<\gamma \mid s_{i k}\left[n-n_{d}\right]=0\right) \\
& =1-Q_{1}\left(0, \frac{1}{\hat{\sigma}_{n}} \sqrt{2} \gamma\right)
\end{aligned}
$$

\section{B. Modulation symbol achievable rate}

The combined signal in (23) passes through the RF chain and the high resolution ADC and thus, the modulation symbol $x_{j}$ can be decoded, for simplicity we drop the time notation in this part. In order to obtain received signal amplitude that does not depend on $x_{j}$ we consider constant amplitude constellation $M$-phaseshift-keying ( $M$-PSK) and thus, $I_{m}$ with the $M$-PSK shaping loss [16] can be expressed as

$$
I_{m}=\sum_{i=1}^{2^{N_{a}}-1} \operatorname{Pr}\left(\mathbf{s}_{i}\right) \sum_{j=1}^{2^{N_{a}}} \operatorname{Pr}\left(\hat{\mathbf{s}}_{j} \mid \mathbf{s}_{i}\right) \times \frac{1}{2} \log _{2}\left(\left.\frac{4 \pi}{e} \mathrm{SINR}\right|_{\mathbf{s}_{\mathbf{i}}, \hat{\mathbf{s}}_{\mathrm{j}}}\right)
$$

$$
\begin{gathered}
\left.\operatorname{SINR}\right|_{\mathbf{s}_{\mathrm{i}}, \hat{\mathbf{s}}_{j}}=\frac{\left(\sum_{k=1}^{N_{a}} s_{i k} \hat{s}_{j k}\right)^{2}}{\max \left(\sum_{k=1}^{N_{a}} \hat{s}_{j k}, 1\right)} \frac{\hat{\alpha} P_{t}}{\hat{\sigma}^{2}} \\
\operatorname{Pr}\left(\hat{\mathbf{s}}_{j} \mid \mathbf{s}_{i}\right)=\prod_{k=1}^{N_{a}} \operatorname{Pr}\left(\begin{array}{c}
\hat{s}_{k}=1 \\
a_{\hat{s}_{j k}=0}^{\gtrless} \gamma
\end{array}\right), \operatorname{Pr}\left(\mathbf{s}_{i}\right)=\frac{1}{2^{N_{a}}}
\end{gathered}
$$

As mmWave channel is spatially sparse and badly conditioned the channel is rank deficient if all receive antennas are selected, so ZF pre-equalizer is not possible unless we ensure full row rank channel. This can be achieved by the smart removal of the receive antennas. We perform the RAS at the BS to maximize the mutual information and then the BS informs the UT about the selected antennas through control channel. The RAS based exhaustive search is computationally complex especially in large MIMO systems. Thus, we propose fast and efficient RAS sequential algorithm based on the QR decomposition [9] as illustrated in Algorithm 1.

In Algorithm 1, the QR decomposition is exploited to sort the best antennas in one step. Then, we add one antenna per iteration to the set $\mathcal{S}$ and stop by decreasing

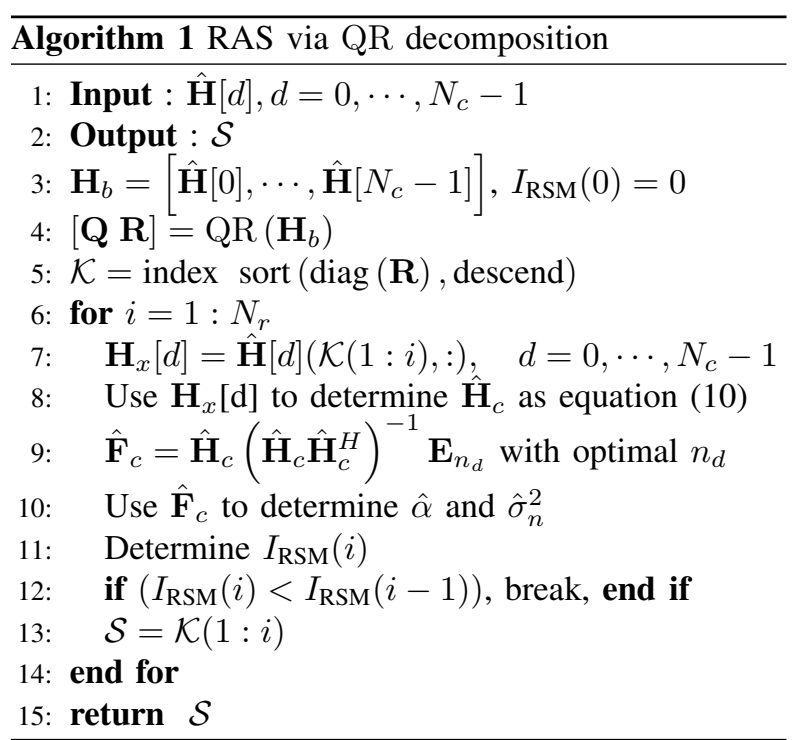

the mutual information. Finally, the set $\mathcal{S}$ includes the selected antennas. The RAS decision is performed at the BS and the UT be informed through zero error control channel. Algorithm 1 is much faster than the exhaustive search as the maximum number of needed iterations at Algorithm 1 is $N_{r}$.

\section{Fully digital OFDM MIMO}

We compare the performance of the proposed energy efficient RSM scheme with the OFDM FD MIMO system. For fair comparison, we assume the CSI is known only at the BS for the FD MIMO system and hence we apply ZF precoder per subcarrier, water-filling power allocation among subcarriers and RAS is performed to maximize the mutual information. We consider $M$ Quadrature-amplitude-modulation ( $M$-QAM) symbols for the FD system with shaping loss $1.53 \mathrm{~dB}$ [17] where the mutual information can be expressed as

$I_{\mathrm{FD}}=\frac{N_{a}}{K+C_{p}} \sum_{k=1}^{K} \log _{2}\left(1+\frac{\frac{P_{k}}{\sigma^{2}} / \frac{\pi e}{6}}{\operatorname{Tr}\left\{\left(\mathbf{H}_{f a}[k] \mathbf{H}_{f a}[k]^{H}\right)^{-1}\right\}}\right)$

where $P_{k}$ is the subcarrier power, $\sum_{k=1}^{K} P_{k}=K P_{t}$, $\mathbf{H}_{f a}[k]$ is the channel after the RAS and $C_{p}$ is the cyclic prefix.

In FD OFDM MIMO, specific precoder is needed for each subcarrier and the UT needs to know the received power per subcarrier to perform amplitude adjustment before detection that increases the DL training overhead when large number of subcarriers are considered. In contrast, the proposed scheme considers single preequalizer and the UT needs one DL training symbol for detection.

\section{Simulation Results}

In this section, we compare the SE and the energy efficiency (EE) of the proposed scheme with FD OFDM system. The EE is defined as the SE per the hardware 


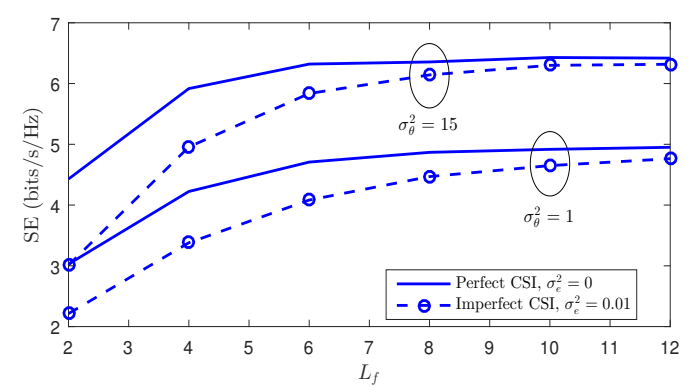

Fig. 3. Spectral efficiency of the proposed RSM scheme with number of pre-equalizer taps, $N_{t}=64, N_{r}=8, \mathrm{SNR}=0 \mathrm{~dB}$ and (average over 1000 realizations).

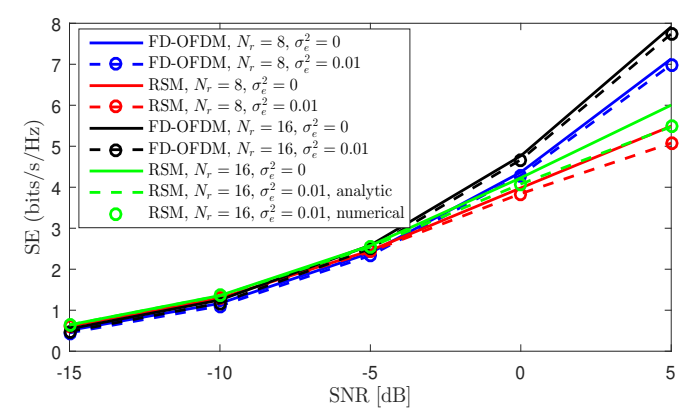

Fig. 4. Spectral efficiency of the proposed RSM scheme compared to FD OFDM MIMO at $N_{t}=32, L_{f}=10$ and (average over 1000 realizations).

power consumption at the UT. We use the power consumption model in [4] with $P_{\text {ref }}=20 \mathrm{~mW}$. In the simulation environment, we consider $C=4, L=16$, $N_{c}=4$ [11], $K=4096, C_{p}=25 \%$ as in IEEE 802.11ad, $g_{l i}$ are i.i.d $\mathcal{C N}(0,1), \tau_{l i} \in\left[0,\left(N_{c}-1\right) T_{s}\right]$ is uniformly distributed, $\left(\tau_{1 i}=\tau_{2 i}=\cdots=\tau_{L i}\right)$, $\left(\phi_{l i}, \theta_{l i}\right)$ have Laplacian distribution with uniform random means $\left(\phi_{i} \in[-\pi / 6, \pi / 6], \theta_{i} \in[-\pi, \pi]\right)$ and angular spreads $\sigma_{\phi}=\sigma_{\theta}=3^{\circ}$ and the signal-to-noiseratio $\left(\mathrm{SNR}=P_{t} / \sigma^{2}\right)$.

TABLE I

AVERAge $N_{a}$ OVER 1000 ITERATIONS AND $\sigma_{e}^{2}=0.01$

\begin{tabular}{|l|c|c|c|c|}
\hline Scheme & -20 & -10 & 0 & 5 \\
\hline Proposed RSM, $N_{r}=8$ & 1 & 1.04 & 2 & 2.4 \\
\hline FD OFDM, $N_{r}=8$ & 1.09 & 1.4 & 2.2 & 2.6 \\
\hline Proposed RSM, $N_{r}=16$ & 1 & 1.07 & 2.2 & 2.7 \\
\hline FD OFDM, $N_{r}=16$ & 1.13 & 1.5 & 2.6 & 3.1 \\
\hline
\end{tabular}

Fig. 3 shows that the SE saturates with the number of the pre-equalizer taps at perfect and imperfect CSI that can be exploited to reduce the hardware complexity of the BS. Channels with wider angular spread allow more ARA, and thus the spatial symbol can convey more information bits that improves the SE.

Fig. 4 and Fig. 5 show that the average and outage SE of the proposed scheme (single RF chain) is comparable with the FD OFDM at perfect and imperfect CSI at the BS. This can be justified as mmWave channels are

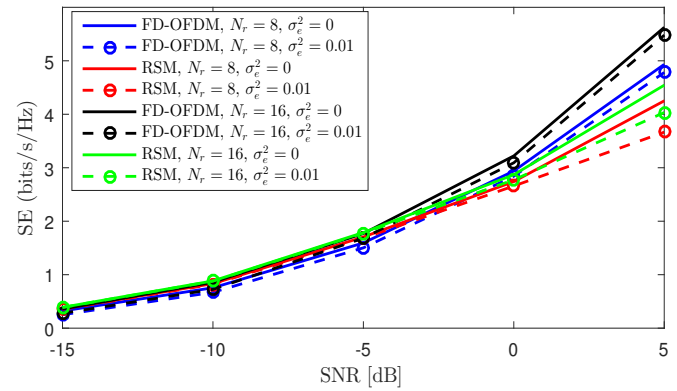

Fig. 5. $10 \%$ Outage spectral efficiency of the proposed RSM scheme compared to FD OFDM MIMO at $N_{t}=32, L_{f}=10$ and (evaluated over 1000 realizations).

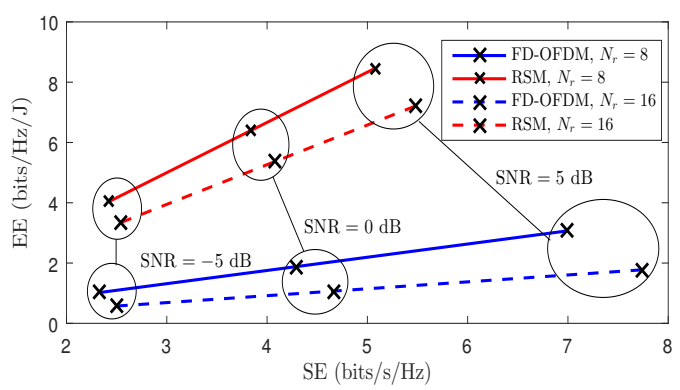

Fig. 6. Spectral efficiency versus energy efficiency trade-off of the proposed RSM scheme compared to FD OFDM MIMO at $N_{t}=32$, $L_{f}=10, \sigma_{e}^{2}=0.01$ and (average over 1000 realizations).

rank deficient and suffers from severe path loss [10] and thus, the ZF precoder is possible with few number of ARA as depicted in Table I. Therefore, the number of transmit symbols per subcarrier for the FD OFDM is less than number of receive antennas.

Table I shows that the number of ARA that maximizes the SE is much less than $N_{r}$ and thus the RAS is substantial with the badly conditioned mmWave channels.

Fig. 6 illustrates that the proposed scheme approaches the SE of the FD OFDM and attains superior energy efficiency because the proposed UT architecture depends on single RF chain and one high resolution ADC as shown in Fig. 1

\section{CONCLUSION}

The proposed wideband single carrier RSM architecture provides an interesting trade-off between EE and SE at the UT. The proposed RSM scheme outperforms the FD MIMO in energy efficiency and approaches the SE. Moreover, in RSM, the BS informs the UT about the detection threshold once per coherence time, on the other hand, the UT in OFDM requires a normalization factor per subcarrier to enable the detection. Not surprisingly, it has been observed that the SE saturates with the number of FIR taps and the minimum number of taps increases with the time spread $N_{c}$. Optimizing the FIR delay $n_{d}$ based on the channel errors is necessary to both combat the intersymbol interference and maximize 
the received signal power. The BS just requires the channel error and receive noise variances to optimize the FIR delay. The RAS is vital for single carrier RSM schemes to make the pre-equalizer possible in rank deficient MIMO channels. Extending the proposed scheme for multi-user system is an ongoing research topic.

\section{REFERENCES}

[1] Cheng-Xiang Wang and et al., "Cellular architecture and key technologies for $5 \mathrm{G}$ wireless communication networks," IEEE Communications Magazine, vol. 52, no. 2, pp. 122-130, Feb. 2014.

[2] T. S. Rappaport and et al., "Millimeter wave mobile communications for 5G cellular:It will work!," IEEE access, vol. 1, pp. 335-349, May 2013.

[3] S. Park, A. Alkhateeb, and R. W. Heath, "Dynamic subarrays for hybrid precoding in wideband mmwave MIMO systems," IEEE Transactions on Wireless Communications, vol. 16, no. 5, pp. 2907-2920, May 2017.

[4] R. Méndez-Rial et al., "Hybrid MIMO architectures for millimeter wave communications: Phase shifters or switches?," IEEE Access, vol. 4, pp. 247-267, Jan. 2016

[5] Lie-Liang Yang, "Transmitter preprocessing aided spatial modulation for multiple-input multiple-output systems," in $73 \mathrm{rd}$ IEEE Vehicular Technology Conference (VTC Spring), pp. 1-5, May 2011.

[6] N. S. Perovic, P. Liu, M. Di Renzo, and A. Springer, "Receive spatial modulation for LOS mmwave communications based on TX beamforming," IEEE Communications Letters, Dec. 2016.

[7] A. Raafat, A. Agustin, and J. Vidal, "Receive spatial modulation for massive MIMO systems," in IEEE Global Communications Conference (GLOBECOM), pp. 1-6, Dec. 2017.
[8] H. Sampath, H. Bolcskei, and A. J. Paulraj, "Pre-equalization for MIMO wireless channels with delay spread," in 52nd IEEE Vehicular Technology Conference (VTC), vol. 3, pp. 1175-1178, Sept. 2000.

[9] Q. Zhou and H. Dai, "Joint antenna selection and link adaptation for MIMO systems," IEEE transactions on vehicular technology, vol. 55, no. 1, pp. 243-255, Jan. 2006.

[10] T. S. Rappaport and et al., "Wideband millimeter-wave propagation measurements and channel models for future wireless communication system design," IEEE Transactions on Coтmunications, vol. 63, no. 9, pp. 3029-3056, Sept. 2015

[11] K. Venugopal, A. Alkhateeb, R. W. Heath, and N. G. Prelcic, "Time-domain channel estimation for wideband millimeter wave systems with hybrid architecture," in IEEE International Conference on Acoustics, Speech and Signal Processing (ICASSP), pp. 6493-6497, March 2017.

[12] S. Rami, W. Tuni, and W. R. Eisenstadt, "Millimeter wave MOSFET amplitude detector," in Topical Meeting on Silicon Monolithic Integrated Circuits in RF Systems (SIRF), pp. 8487, Jan. 2010

[13] T. M. Cover and J. A. Thomas, Elements of information theory, John Wiley \& Sons, 2012.

[14] A. Raafat, A. Agustin, and J. Vidal, "Receive antenna selection and hybrid precoding for receive spatial modulation in massive MIMO systems," in IEEE International ITG Workshop on Smart Antennas (WSA), March. 2018

[15] F. Chapeau-Blondeau, "Noise-enhanced capacity via stochastic resonance in an asymmetric binary channel," Physical Review E, vol. 55, no. 2, pp. 2016, Feb. 1997.

[16] R. E. Blahut, Principles and practice of information theory, Addison-Wesley Longman Publishing Co., Inc., 1987.

[17] G. D. Forney and G. Ungerboeck, "Modulation and coding for linear Gaussian channels," IEEE Transactions on Information Theory, vol. 44, no. 6, pp. 2384-2415, Oct. 1998 